\title{
Deaf access for Deaf people
}

The translation of the television news from English to British Sign Language

Christopher Stone (christopher.stone@ucl.ac.uk)

The DCAL Centre, University College London

\begin{abstract}
This paper explores the notion of a Deaf translation norm and its use in the rendering of English mainstream television broadcast news into British Sign Language (BSL). The Deaf translation norm incorporates the community identity and fluency of the translator/interpreter (T/I). Historically this is a role that Deaf bilinguals have undertaken and in part involves the higher level of agency that the T/I exerts within the situation. In present day this differs from a historic role now that the translation event happens in the public sphere rather than within the community. To ensure that the Deaf audience has an optimally relevant BSL text to watch and understand, the Deaf translation norm incorporates enrichments and impoverishments into the BSL text according to their understanding of the English text and the video footage that is shown simultaneously on screen. This creates a BSL text that utilises the multimedia environment to reduce the cognitive effort of the Deaf audience.
\end{abstract}

\section{The Deaf Community}

Convention has arisen that a person who has a Deaf cultural identity and is audiologically deaf is called 'Deaf' (Senghas \& Monaghan, 2002) and this distinction has been made within the community for several hundred years (Ladd, 1998).

Membership of the Deaf community in the past has predominantly been due to being born deaf or losing hearing at an early age so that no sense of loss is felt. Cultural identity was then forged by attending schools for the deaf in early life and Deaf clubs throughout the rest of life. As Ladd (2003: 44) says:

This traditional community therefore consists of Deaf people who attended Deaf schools and met either in Deaf clubs or at other Deaf social activities.

In more recent years membership of the Deaf community has been defined by Baker and Padden as follows

The most useful basic factor determining who is a member of the deaf community seems to be what is called 'attitudinal deafness'. This occurs when a person 
identifies him/herself as a member of the deaf community, and other members accept that person as part of the community (Baker \& Padden, 1978:4).

The attitudinal deafness discussed by Padden clearly refers to the traditional community discussed in Ladd. As discussed by Ladd (2003:42) the community is strengthened by $90 \%$ endogamous marriage. Five percent of Deaf people born deaf have Deaf parents and a further five percent have one parent who is Deaf (Kyle \& Allsop, 1982; Kyle \& Woll, 1985). The extent to which these families have many generations of Deaf is unknown, but there are known cases of one Deaf family having documented seven generations (with a grand child resulting in eight generations) in Britain (Taylor, 1998) although there are anecdotal stories from this family suggesting there are ten generations (with a grand child being the eleventh).

These multi-generational Deaf people are seen as the core members of the Deaf community. They are the ones who have experienced life, at least within the home, as a Deaf haven from a hearing world. As the guardians of sign languages, Deaf history and culture there is an expectation that they will preserve and pass on Deaf ways of being in the world (Padden \& Humphries, 1988). Taking my Deaf T/Is from this group enables me to explore what a Deaf translation might be like if it were not for the 'hearing' institutional barriers that the T/Is face in the news studio.

The historic Deaf community is described as a collective community (Ladd, 2003), and therefore allegiance is to the minority community rather than the individualistic values of the (mainstream) 'hearing' community. Although this can be contested, and arguably the present day Deaf community is a heterogeneous community (Skelton \& Valentine, 2003a, 2003b), the Deaf T/Is in this study, from multigenerational Deaf families, adhere to traditional notions of collective identities (Smith, 1996).

\section{The research}

The research draws on data from ethnographic interviews (Carmel \& Monaghan, 1991; Cook \& Crang, 1995; Spindler \& Spindler, 1992; Spradley, 1979) of five Deaf 
T/Is (all of whom chose pseudonyms ${ }^{1}$ ) who regularly work in broadcast television news. All five have graduate level training in linguistics and experience both in working as and training interpreters. In an attempt to ensure that the research was Deaf-led or Deafhood (Ladd, 2003) informed ${ }^{2}$ (whilst Deafhood-informed ensures that research is undertaken in a Deaf culturally sensitive way it does not have to be carried out by Deaf people) semi-structured interviews of Deaf informants were used to generate grounded theories (Strauss \& Corbin, 1990). The research aimed to be Deaf-led and to explore what was deemed to be relevant to Deaf T/Is (the informants are quote throughout this article) and as such no hearing interpreters were interviewed.

\section{The British context for BSL on television}

Under the Broadcasting Act $1996^{3}$, broadcasters are obliged to provide five percent of programming by $2005^{4}$, either presented in, or translated into, sign language. This does not mean that the broadcasters feel political affinity with the provision of BSL access but rather see it as a legal obligation, like other equality laws, that needs to be fulfilled (Squires, 2004). There are institutional constraints on how T/Is work in broadcast media similar to the institutional constraints that interpreters face in other domains. Inghilleri (2003) considers interpreters within an asylum-seeking context. She discusses the need for the interpreters to ensure the believability of the interviewee by constructing their narrative as believable to the interviewer. This requires the interviewee to be perceived as a victim by the target audience within target cultural norms rather than constructing the story in a way that is believable and valid in the source language and culture.

This is mirrored by BSL T/Is working within a broadcasting context, although we see that the power dynamic is flowing in a different direction, from majority to minority audience. In the context of asylum seeker interviews, where the person's history must

\footnotetext{
1 These were Clark, Georgina, Kim, Kat and Rebecca

${ }^{2}$ An idea mooted by Turner and recently explored at REMEDES 2004.

${ }^{3}$ http://www.hmso.gov.uk/acts/acts1996/96055--h.htm\#20

${ }^{4}$ http://www.ofcom.org.uk/tv/ifi/codes/ctas/\#content
} 
be seen as valid within a majority context from a minority context, there is no room for any of Venuti's (1998) suggestions for a translation preserving difference - rather there is the need for domestication and trying to ensure the naturalness of the TL ${ }^{5}$. This parallels the situation in the broadcasting context for BSL T/Is.

In this context, the audience not only comprises the Deaf target audience, but also a mainstream audience that sees the in-vision T/Is. The T/Is must construct a product that is not only acceptable to the Deaf audience but also to the mainstream audience in terms of cultural expectation (Woll, 2000) such as fewer grammatical facial movements and a speed perceived by the mainstream audience (themselves ignorant of the TL) to be appropriate in this medium.

Interpreted/translated television news in the UK is broadcast in two different formats, headline news summaries or weekly news reviews, with the T/Is finding themselves in two different situations. In the first situation, found in news week review broadcasts, the Deaf T/Is (as 'presenters') are acknowledged as being present and introduce themselves and the hearing anchor. They present some of the news stories and perhaps engage in some interaction with the hearing anchor in BSL. The Deaf T/ Is in this role have had access to the script beforehand, have prepared how they will present this information, and are following the newsreader's autocue when presenting.

In the second situation, the Deaf T/Is are there to present the news stories of a reporter on location or with video footage and a voiceover. The Deaf T/Is have again had sight of the script beforehand and prepared their presentation of the information. The Deaf T/Is will have been introduced by the anchor (hearing only, if during a news headlines format; hearing and Deaf during a news week review format) and will not be present other than to present the information in the news story.

In both situations the Deaf T/Is read an English script, prepare a BSL translation of that script and then read the English autocue whilst presenting their BSL version of

\footnotetext{
${ }^{5}$ Target Language in this case BSL
} 
the information. The situations differ, though, in the status of the Deaf T/I: in the first situation they are co-presenting information with the newsreader (in the anchor position) and in the second situation they are re-presenting pre-recorded news from a reporter.

When the Deaf T/Is are in the anchor position, the hearing anchors co-presents information with their Deaf anchor colleagues from the same autocue and co-operate with each other so that the needs of the Deaf audience are taken into account, especially the speed that information is presented in spoken English. The anchors work together creating greater space and time for the Deaf T/Is to make translation additions to the news stories so that the translation is relevant to the audience. As one of the informants says:

I know that the news team they try to give me time, lengthen it per story, often, it depends on the producer, if they know when to slow done or give me longer, for some it's short and that's that, that can happen but it's rare (Kim)

When the Deaf T/Is are translating reports, however, the English voice-over is prerecorded and so this imposes a fixed time within which the translation must occur.

The space created whilst the Deaf T/Is are a 'presenter' also allows for the Deaf T/I anchor to introduce things, e.g. the names of protagonists and their ages. This can then make things easier for the Deaf T/Is translating the reports, since this information no longer needs to be included. The Deaf T/I anchors greet the audience but this is something that the Deaf T/Is as translators also try to do in any case, to build a good rapport with the audience. This act of being present in the second situation, however, is brief when compared with the highly present nature of the anchor Deaf T/Is.

The Deaf T/Is do not see the task of 'anchor' and 'signer' differently in terms of an act of translation, but they are aware that they are labelled differently according to how they appear on screen. Similarly, whilst the Deaf T/Is know that they are translating the English, they are doing this within a mainstream news programme and see themselves as newsreaders for the Deaf community. They are members of this 
community and one of the highest things valued by the Deaf T/Is is that they produce a comprehensible TL for their target audience.

\section{The historic roots}

The Deaf T/Is understand the role that they undertake in television to be a role that bilingual Deaf people have undertaken for many years. Since the inception of Deaf clubs, the bilingual Deaf person has supported the community by translating letters, newspapers and information generally to semi-lingual (Hinnenkamp, 2005; SkutnabbKangas, 1981) and monolingual Deaf people. This still happens today. This is considered part of one's responsibility to the community, and is an example of the reciprocal sharing of skills within the community's collectivist culture (Smith, 1996). The rendering of broadcast news is the first time that this role has emerged into the public domain.

It is worth bearing in mind that the Deaf community is not able to commission which news will be translated. Unlike when a member of the community takes their letter, or a newspaper article to be translated, the news being translated is that chosen by hearing, non-Deaf, producers and journalists. The Deaf bilinguals exercised their judgement in informing the semi-lingual and monolingual Deaf people about events happening in mainstream society. So to some extent the Deaf bilinguals acted as gatekeepers to the information being passed on to their community (Ladd, 2003; Vuorinen, 1995), in that if they did not think that the information was relevant then this information was not passed onto the community.

This is where the new role differs because neither the Deaf community nor the Deaf T/Is choose the news that is to be rendered for the Deaf community. And some of the news scripts that the Deaf T/Is are asked to work with are not judged, by the Deaf T/ Is, to be of interest (or relevant) to the Deaf audience: 
When using the script as a guide, the Deaf T/Is move towards considering themselves as bilingual newsreaders, both when they are anchors and when they are 'signers'. They do not consider themselves knowers of the information per se, but as deliverers of information approaching that information delivery from a translation perspective. One of the Deaf T/I informants states:

If the text is clear then I can produce the information clearly, if the information is complex I don't repeat it complexly as the audience would not understand it, I need to make changes, what it means exactly, then I ignore the script, change the delivery so that I say exactly what the story means, then add the details and build it up so that it matches the meaning of the script. (Clark)

This action fulfils a role that historically proficient English readers would have had in the Deaf community.

\begin{abstract}
first interpreter been years ago ... always have Deaf "interpreter" always ... I don't mean for example maybe hearing that then straight away sign it to Deaf, no I mean what's going on in society, tell Deaf that interesting, that, Deaf oh wow, interesting that, we've always had that, to me that's part of interpreting, like newspaper morning been read or TV been read then let people know, oh that was really bad, you know, and explain that Deaf really interested, that always have, or Deaf have letter, know someone excellent English, ask them to explain, always have but that has always been in the community rather than open and public (Clark)
\end{abstract}

The historic role informs the translation style that the Deaf T/Is use; it is the precursor of the present day Deaf translation norm. The translation act needs to be relevant to the constructed audience (Ruuskanen, 1996):

Maybe hearing in-vision I look between the subtitles and the in-vision interpreters and I think yes, but I would call it hearing structure, you don't want that, the audience it will fly over their heads, the subtitles are enough, what is the in-vision interpreter there for? It's a waste them being there, I always say that the subtitles and the invision interpreter should be really different, they should be different but the information that you understand for them should be the same, if they are similar then what is the in-vision interpreter there for? (Clark) 
So that the viewer experiences a parallel understanding of the $\mathrm{SL}^{6}$ information, the

TL draws upon the visual information that is being seen on the screen.

\begin{abstract}
I have to create a clear mental picture for them, which means that I have to try to digest the information and then think how I can sign output which gives them a clear mental picture, by creating a clear picture for myself, then think that's it, I want the Deaf audience to have the same mental picture as me, not sign it so that the Deaf people have to build their own picture bit by bit (Georgina)
\end{abstract}

By pointing towards relevant visual information and using this to inform some of the grammatical decisions (such as the visual shape and size of objects (Supalla, 1986)) this ensure that the TL information is maximally relevant (Gutt, 1998; Setton, 1999; Sperber \& Wilson, 1986, 1995; Wilson \& Sperber, 2002) to the audience.

\title{
Relevance to the audience
}

The Deaf T/Is (aim to) use the English autocue interpretively (Gutt, 1998), such that they are not creating a 'faithful' TL, but rather a TL that is optimally relevant to the Deaf audience. This involves including information that will have appeared either earlier during the week (for news week review programmes) or earlier in the broadcast (for news headlines). As information is the key to the news broadcast, that is given primacy over other parts of the message such as the metanotative qualities ${ }^{7}$ (Isham, 1984) of the newsreader.

Clark (one of the informants) questions some accepted notions of the skopos (Hatim, 2001:73; Vermeer, 2000) or goal of the translation and how the SL is to be used by the in-vision interpreter:

\footnotetext{
I know that in training interpreters are told not to make additions to what someone says if they are monotonous or boring then you need to match their style to add things so that the audience think it's great ... that's a dilemma should you match the style of the person or just deliver the information not give their style over that's hard ... there is no easy answer if people specifically ask you to portray the character of
}

\footnotetext{
6 Source Language in this case English

7 These are the non-content characteristics that influence or determine a person's overall impressions of the speaker (Isham, 1984: 119)
} 
the person then you are clear but I don't think that that is specifically needed in this situation what is important is information (Clark)

In other words, the information in the script is understood and re-presented in a way that is pragmatically understood by the audience. The information that has been broadcast previously is used so that the TL can be re-edited to include both enrichments and impoverishments (Sequeiros, 1998, 2002); whatever is required to minimise the effort on the part of the constructed audience (Ruuskanen, 1996).

In some instances the implicatures that are constructed within the BSL text are different from the implicatures of the English text. This ought to be avoided: the invision T/I needs to ensure that the language is used in such a way that it points towards the same inferred meaning as the ST; the BSL needs to be presented as an ostensive utterance in an appropriate way. The TL interpretive utterance will then gain greater resemblance to the SL original (Gutt, 1998).

The news headlines summary has the least amount of time available for a relevant BSL text. In order to create the most relevant BSL text, the Deaf T/Is therefore like to use the information already available to the Deaf audience via the subtitles and the visual footage in the more comprehensive news previously broadcast to create the most relevant BSL text. If they are to use the additional information previously broadcast to judge how the BSL can be made maximally relevant to the constructed audience, the Deaf T/Is need more information than is contained in the English news summary. One of the informants describes this below:

there is background information on the news, the news from 6 to 25 past has lots of information and I read that to find out what they are talking about, then look at my script, much reduced information, if I feel it can be delivered as is I do it, if there is one word that is difficult I can take information from the larger script add it so that it has the same meaning with that background information, I only started doing this recently and Clark is the same, it's good restructuring and adding background information so that it is clear ... really for a Deaf audience it's only 30 seconds, what I feel is appropriate information from the larger script, I can't tell the script writers 
that their summary is poor so I add information, just one or two pieces so it's clear (Rebecca)

So the Deaf T/Is not only enrich the SL, but also act as a journalist to the extent that they appropriately edit the larger story for the headlines summary. This happens within the constraints of the time and visual information that is being shown on the screen. It also fulfils the agency that they believe they should use to fulfil the Deaf translation norm.

\section{Deaf translator role to interpreter role}

One of the news review programmes changed its format slightly throughout the course of the research. Rather than just being a review of the week's news, the programme also included a preview of television programmes that were to be broadcast in the coming week. The floor manager also changed with the new format. The previous floor manager had worked well with the Deaf T/Is, so that they felt accepted as part of the team. The new floor manager saw the Deaf T/Is as outside of the team so that the Deaf T/Is felt that there was little acceptance of them as part of the newsreading team.

The Deaf T/Is felt that after filming a section in the new format, their agency was limited and changes could not be made. In the previous style programme, if the Deaf T/Is were not satisfied with their work, there was an opportunity for the section to be re-filmed. In the eyes of the Deaf T/Is this lack of agency was compounded by the format of the new style programme. It comprised many clips and short pieces of information, combined with video footage. This style of programming gave the Deaf $\mathrm{T} / \mathrm{Is}$ little chance to re-structure or re-order the news and programme information. One of the Deaf T/Is, Kat, reported to me that they felt like a HEARING INTERPRETER ${ }^{8}$. I raised this issue with the other informants and they agreed with this sentiment.

\footnotetext{
${ }^{8}$ The BSL signs are glossed using upper-case lettering (e.g. HEARING).
} 
This seems to indicate that for the Deaf T/Is this confined role is more indicative of the 'hearing'(or non-Deaf) interpreter. The Deaf T/Is want more agency (Inghilleri, 2003; Rudvin, 2002, 2004) within the broadcast news. The hearing interpreter, from the Deaf T/Is' perspective, collude with the institution by not demanding greater time and space to include cultural and linguistic adjustments and enrichments in the TL.

The preliminary norm (Toury, 1978/revised 1995) is one where Deaf T/Is are not able to choose the news items that are translated. In spite of this, the Deaf T/Is feel that in some contexts they are given enough agency to create some idea of ownership of the process and the information.

on Newsweek or the headlines we are always talking about changing to match the audience ... we have to find out what the background information is, the script may not be clear, and so we need the background information so that we can put those in and sign it so that it is understood by the audience, we need to put in cultural information (Clark)

When this agency is denied them, they feel used by the institution in perpetuating mainstream values.

some of the reports are really coming from hearing culture but we have to deliver the information we can't edit it or change it but just deliver it, sometimes they are trying to be funny and I would question whether the Deaf audience gets it or not, because it is a hearing thing, but I can't do anything about that that's beyond my control (Rebecca)

This conflicts with the Deaf T/Is' historic and cultural rationale for being employed. Accordingly, the Deaf T/Is are unable to fulfil the skopos of the translation as they see it. The Deaf T/Is are not able to adhere to their Deaf translation norm. This Deaf translation norm is, however, a value that is shared by the Deaf T/Is and not necessarily identified by nor accommodated by the news broadcast institutions. 


\section{Presence}

The Deaf T/Is see themselves as being more 'present' (Stolze, 2004) when delivering the news information in BSL when compared with the hearing interpreters. They believe that this greater presence is a core part of the Deaf translation norm

The Deaf T/Is are re-presenting the information:

we look at the whole thing, take it on board, chew on it, conceptualise the whole thing, what it is, then take on board that conceptualisation and present that in BSL (Georgina)

So the information is not 'just' translated or interpreted into BSL but presented to the audience. There is no notion of neutrality embodied in that presentation.

This presence means that the Deaf T/Is see themselves more closely in the role of bilingual reporter/journalist than interpreter. There is some ownership of the information and the way that this is presented to the Deaf audience.

\footnotetext{
simultaneous interpreting just goes on and on and on, you focus on processing and editing and reformulating information, and as you said relay the information, so I feel that for it to become Deaf you have to be in it, for it to become a full translation it has to be consecutive ... it is more BSL when you sign ... you have the information in your head and sign it clearly to the Deaf person, that creates instant rapport, with simultaneous you are out of the information (Kim)

if you are reading written materials understanding them and then translating them for an audience that is still consecutive because you take it section by section (Kim)
}

So by not undertaking an online process, the Deaf T/Is are able to have greater rapport with their constructed audience. And this is how the Deaf T/Is create presence in their renderings.

This presence occurs more readily when the news stories are deemed relevant by the Deaf T/Is. When the news stories are viewed as inappropriate for a Deaf audience, then the Deaf T/Is move into functioning in the way they perceive the hearing 
interpreters to work. The Deaf T/Is become more detached from the information and less present when delivering the news story.

\section{Translation Performance}

One of the things raised about the translation of the English into BSL is that there is still a performance factor to this task. Unlike the translation of two written languages, with the translation of a written language into an unwritten language there is a limit to the extent to which the TL can be edited. The longer the news story, the less the Deaf T/Is are able to construct a fully edited TL. And even with short news stories, if it is a live broadcast, once the broadcast has started any performance errors need to be corrected 'online'. This is also true to a lesser extent with pre-recorded broadcasts, if the Deaf T/Is are seen as part of the team and allowed to re-record stories.

This seems to be related to the nature of the interaction between having a prepared translation that is memorised and a scrolling autocue that acts as a prompt but is not a separately prepared autocue containing a gloss. The SL is still written English, but a translation has been mentally prepared. As such there is a translation performance issue because BSL is an unwritten language.

I feel the task is interpreting when, if I have been mentally processing then that is interpreting, me if I have been processing, like TV news you are right, I feel it is translation, but I am still processing, reading the autocue, processing and changing it into sign, that means processing, so I feel TV news is a mixture of interpreting and translating (Kim)

The English on the autocue is the same as that used by the newsreader and any notes in English or glosses are not prepared on a separate autocue for the Deaf T/Is. In this way the interaction differs from consecutive interpreting, where notes of the SL can be written down prior to reformulation. The reformulation is undertaken by the Deaf T/Is beforehand, but if this cannot be remembered due to the live nature of the translation, then the English autocue will still interfere with the TL production when being broadcast. 
if you do not remember the translation, the autocue is fixed ... that's difficult, sometimes I remember I can sign sometimes and not follow the autocue, it interfere influence our [Deaf T/Is] structure (Georgina)

This is compounded by the lack of control that the Deaf T/Is have in a live broadcast when compared with interpreting in a meeting. The meeting can be stopped and clarifications can be made, but this is not possible during a live television broadcast. In a pre-recorded programme this is also something that comes into play.

lots feel play safe stick to English order because it's live if it were pre-recorded it's better you have more control (Kat)

The idea of translation performance is useful when thinking about translating BSL.

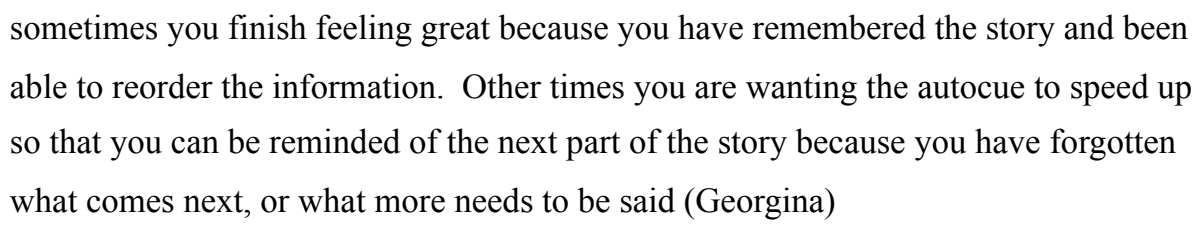

This is analogous to language competence and performance (Akmajian, 1995) where internal language competence cannot be judged by performance errors. A native language user can have intuitions about the grammaticality of a sentence whilst making mistakes in production because performance errors occur. This happens in the case of translating oral languages, although signed languages maybe the only example of unwritten languages being translated, when due to the performance of the translation errors occur. So, whilst the translator can construct an internal 'mental' translation using their translation competence, and judge the grammaticality of another translation, errors can still occur in the translations they produce themselves.

Clark is really skilled at presenting sometimes you can tell if the script was given to him last minute but most of the time it doesn't bother him and he signs naturally Clark is good and one of the few compared to others and we know he can read and understand and memorise the information and you know he is skilled at translating he doesn't allow the order to influence him at all and so he just delivers the information sometimes he is reading but that doesn't affect his output but sometimes you catch 
him hesitating and you know that it is last minute information that he hasn't had a chance to fully digest but when he does have time he is great (Kim)

Interestingly, when a story is repeated and the Deaf T/Is become more familiar with the information they are more able to act as information deliverers.

sometimes me nothing mentally processing, nothing sometimes, I read the script, translate it but I already know the information, I don't need to think, like if it is a repeated news story, like Soham the small girls murdered, that was repeated so I did not need to process (Kim)

The less familiar the information is to the Deaf T/Is, the more likely they are to perform in a way that is perceived to be like a hearing interpreter.

\footnotetext{
I feel I behaviour more like an interpreter when doing simultaneous interpreting ${ }^{9}$, watching the SL and signing the output is not mine, if I wait until I understand it and then tell the Deaf person what I understand that's it consecutive, that's Deaf, simultaneous is just passing on information (Kim)
}

Here the information is unscripted and so less familiar to the Deaf T/I than a scripted piece. Even though an interpreter will rely on prediction skills and will prepare for a job, there is still a different level of known information in a fully scripted SL to be translated when compared with a live meeting.

When space is given for the translation to be made so that a real connection can be made with the audience that is when one acts according to the Deaf translation norm. This relates to the idea that there have always been information sharers and their responsibility within the community is to ensure that the Deaf community members understand the information. In fact the notion of simultaneous interpreting clearly is not conducive with a Deaf translation style, and does not form part of the Deaf translation norm. 
I feel that it is hearing their rules, following this that and the other theirs hearing, if Deaf it would not be like that, Deaf rules are different (Kat)

Hearing interpreters are deemed to follow their professional rules, which are seen as mainstream led and not Deaf culturally sensitive, rather than being involved in the information and involved in ensuring that the message is understood.

theirs, I feel interpreting hearing theirs, I feel the rules, how to behave, stiff, how to sign, all these rules, they are trained, like that, I feel that is there (Kat)

the information is signed outside of the interpreter (Georgina)

This last quotation regards the language style that the hearing interpreters are seen to adopt by the Deaf T/Is. Rather than being involved in a scene and using either constructed dialogue or constructed action ${ }^{10}$ the hearing interpreters use other devices (Quinto-Pozos, in press). This lack of involvement, described as empathetic and participatory by Ong (1982), compounds the lack of language adjustment on the part of the hearing interpreters.

It is important to say at this juncture that this neither describes all hearing interpreters (or Deaf T/Is as contrasting with the hearing interpreters), nor describes the needs of all of the Deaf community.

the point is interpreters can hear the information and relay it, Deaf can relay the information too, but in a Deaf way, the Deaf can follow, suits Deaf way but if they don't that becomes like interpreting (Kat)

The comments of the Deaf T/Is are couched within the notion of the differences between Deaf T/Is and hearing interpreters where those differences could be seen as problematic. There are hearing interpreters who are able to take control of communicative situations (and, conversely, Deaf interpreters who are not). There are those in the community who are less empowered because of their language usage and

\footnotetext{
10 This is the sign language equivalent of reported speech where the person signing either becomes the person and reports their speech or enacts their actions
} 
need greater action on the part of the interpreter in terms of ensuring that the message is understood. In this situation, the Deaf T/Is describe the need for a Deaf T/I to be brought in to act as an intermediary. This greater action to ensure that the message is understood is something that the Deaf T/Is also take within the media. To that extent the Deaf translation norm is not just about doing a 'good' translation but creating a TL that is understood.

Again lots of the time we all think that we are aiming for people like us and those that we mix with, often we forget grass roots Deaf people and it's worse for grass roots Deaf people because the newspaper isn't accessible because it's in English, the TV subtitles don't provide access, so really the programme is for them not to others who have access, they have it already (Kat)

\section{Identity}

The Deaf T/Is are well aware of their core membership in the community. This comes from being born into and growing up in the Deaf community. The Deaf T/Is still socialise with the Deaf community, as that is their home community. This reinforces the Deaf T/Is identity and adeptness at modifying their language so as to be understood by other members of their community.

The Deaf interpreters make decisions in relation to their translations according to how they construct themselves as core community members who regularly interact with the Deaf community.

Deaf people identify those [language] factors without thinking about it and adjust their language without training, they just adjust their language because this the community they mix in, so they just know how to adjust their language (Clark)

When the Deaf T/Is categorise 'hearing' (non-Deaf) interpreters as non-members of the Deaf community, they then do not want to license these non-Deaf interpreters to make similar decisions with respect to the TL. 
[hearing] interpreters dip in and out of the [Deaf] community, if a hearing interpreter was deeply involved in the community all the time then maybe they could take on board all of these factors perhaps, but most interpreters now a days dip in and out, that's it, they work in the community and then leave, it's rare that interpreters now a days are like those in the past, that was different, they were part of the community and socialised within it, it's different now (Clark)

as I read down the text I pick out what my mental picture which is automatically the same as Deaf people but those interpreting read the information and try to change the order but the picture they have isn't right (Kim)

The aim of the Deaf translation norm is to remove traces of the SL so that the TL audience perceive the text as their own.

\begin{abstract}
No they are different I don't know how hearing interpreters create their mental pictures I can talk about Deaf interpreters as one of them or how I understand/receive information from hearing interpreters so what I normally do is read the piece of text and try to think of the Deaf audience I have to create a clear mental picture for them which means that I have to try to digest the information and then think how I can sign output which gives them a clear mental picture by creating a clear picture for myself then think that's it I want the Deaf audience to have the same mental picture as me not sign it so that the Deaf people have to build their own picture bit by bit
\end{abstract} (Georgina)

In this way the Deaf T/Is use this space on televised news broadcasts to create a Deaf space.

maybe Deaf read the script, have understanding of it, they have their experience, their background, their own, this they relate to the script, and add cultural knowledge, maybe hearing read the English autocue or hear it, they think, oh yes, I know, before, have over there, relate to autocue, I feel hearing maybe have limit, through have to be Deaf to empathise, same Deaf have limit through English script (Kat)

Similarly it is reported by the Deaf T/I informants that there are some Deaf T/Is using hearing interpreters as role models.

Hearing interpreters should copy Deaf interpreters but it's happening the other way round Deaf interpreters are copying hearing interpreters why is that? (Kat) 
This seems to imply that the Deaf interpreters are not following the Deaf translation norm, but rather using models that are used by hearing interpreters. It is a complex matter, because hearing interpreters are the norm in most situations other than television. The hearing interpreters can interact with the mainstream majority and theirs is clearly the dominant discourse within the sign language interpreting profession.

The Deaf translation norm is something that has evolved and developed within the Deaf community. It is this home grown quality that is important in creating a Deaf space on television. A translation that the Deaf constructed audience identify with.

\footnotetext{
although there are some Deaf in-vision here and elsewhere the rest are not suitable, they are not native users of BSL, sign with bad articulation, fingerspelling, not clear, shame that group not better, more groups then can compare, at the moment too many learn BSL late, become in-vision, it's a mess, some hearing interpreters better than them, yes, (Rebecca)

not criticism but hearing interpreters good but not have some specific skills, like really hearing will never have those skills, same Deaf with never have some skills that hearing have never, I think it's possible for us impossible for them, through influence from sound, impossible, way of thinking, impossible, do your best fine, but Deaf natural, grow up natural, comma full-stop from head, you tell me I've been I don't know why, not taught in school, how to move head nothing, just using language naturally have head movement there natural (Rebecca)
}

So notions of naturalness are an important part of the Deaf translation norm and that comes from growing up in the community and being natively fluent in the language. Similarly, it would appear that this is not something that hearing interpreters can achieve, although there are aspects of this translation norm that can be achieved. If an interpreter is Deaf (hearing), then they have grown up in the community and potentially have a high degree of fluency, but cannot reduce the influence that hearing sound has on their way of thinking. 


\section{Institutional limits}

In striving to achieve a TL product that is understood with minimal additional processing effort on the part of the audience, when compared with BSL that is not a translation, the Deaf T/Is still have to work within the limits of the organisation for which they are working.

I'm in their hearing news, me in hearing, their news, I do not make big changes to Deaf-cultural their way, me no, at first when learning it was a struggle, now I do not always sign in a strong Deaf way, really should and can float between strong Deaf and less strong, me not so strong Deaf, I have thought why, of course it is because it is hearing news, their structure, that means how can I radically change it to Deaf's if it is based on English, based on hearing structure, it would have to be the other way round with me sitting at the desk an the hearing person standing at the side, that would be Deaf (Kim)

The Deaf audience relies solely on the ability of the Deaf T/Is to provide access to the hearing news, without any support during the broadcast from the hearing institution. The autocue is the feed that the hearing newsreader has and an additional camera with autocue, with a different BSL structured script, could easily be set up for the Deaf T/ Is. This is not necessarily the preferred way of working for the Deaf T/Is, but it is the minimum that the television studio could do to accommodate Deaf T/Is to perform a live translation of the news.

It would be interesting to interview the television newsreaders and others within the institution to have a greater understanding of their motivations for having Deaf T/Is on the news. Now that there is legislation that a specified percentage of broadcast television is mandated to have BSL interpretation or be a BSL based programme, as well as the official recognition of BSL in 2003, this may influence their behaviour in accommodating the Deaf T/Is. The question that this raises is whether there is a genuine commitment to access for the Deaf community with BSL as a first or preferred language, or if the prime motivation in having Deaf T/Is is to satisfy legislative obligations and that the Deaf T/Is must work within the limits set by the institution. 


\section{References}

Akmajian, A. (1995). Linguistics: an introduction to language and communication (4th ed.). Cambridge, Mass. ; London: MIT Press.

Baker, C., \& Padden, C. (1978). American Sign Language. A look at its history, structure, and community. Silver Spring: Linstok Press.

Carmel, S., \& Monaghan, L. (1991). An introduction to ethnographic work in deaf communities. Sign language studies, 73.

Cook, I., \& Crang, M. (1995). Doing Ethnographies (Vol. No 58 Quantitative): Environmental publications.

Gutt, E.-A. (1991). Translation and relevance: cognition and context. Oxford, UK ; Cambridge, Mass., USA: B. Blackwell.

Gutt, E.-A. (1998). Pragmatic aspects of translation: some relevance-theory observations. In L. Hickey (Ed.), The pragmatics of translation. Clevedon: Multilingual Matters Ltd.

Hatim, B. (2001). Teaching and researching translation. Harlow: Longman.

Hinnenkamp, V. (2005). Semilingualism, double monolingualism and blurred genres on (not) speaking a legitimate language. Journal of Social Science Education(1).

Inghilleri, M. (2003). Habitus, field and discourse: interpreting as socially situated activity. Target, 15(2), 243 - 268.

Isham, W. P. (1984). The Role of Message Analysis in Interpretation. Paper presented at the RID Conference Interpreting: The Art of Cross Cultural Mediation.

Jääskeläinen, R., \& Tirkkonen-Condit, S. (2000). Tapping and mapping the processes of translation and interpreting. Paper presented at the Benjamins translation library ; v. 37, Amsterdam ; Philadelphia.

Kyle, J., \& Allsop, L. (1982). Deaf people and the community: final report to Nuffield Foundation. Bristol: Bristol University, School of Education.

Kyle, J., \& Woll, B. (1985). Sign language : the study of deaf people and their language. Cambridge ; New York: Cambridge University Press.

Ladd, P. (1998). In search of Deafhood towards an understanding of British Deaf culture. Unpublished Ph. D., University of Bristol, Bristol. 
Ladd, P. (2003). In search of Deafhood. Clevedon: Multilingual Matters.

Padden, C., \& Humphries, T. (1988). Deaf in America. Cambridge, MA: Harvard University Press.

Quinto-Pozos, D. (in press). Can constructed action be considered obligatory? Lingua.

Rudvin, M. (2002). How neutral is 'neutral'? Issues in interaction and participation in community interpreting. In M. Viezzi \& G. Garzone (Eds.), Perspectives on Interpreting (pp. 217 - 233). Bologna: CLUEB.

Rudvin, M. (2004). Cross-cultural dynamics in community interpreting. Troubleshooting. In Claims, changes and challenges in translation studies: selected contributions from the EST congress (pp. 271 - 283).

Ruuskanen, D. D. K. (1996). The effect of pragmatic factors on the definition of equivalence in translation. Language sciences, 18(3-4), 883-895.

Senghas, R., \& Monaghan, L. (2002). Sign of their times: Deaf communities and the culture of language. Annual Review of Anthropology, 31, 69-97.

Sequeiros, X. R. (1998). Interlingual impoverishment in translation. Bulletin of Hispanic studies, 75, 145-157.

Sequeiros, X. R. (2002). Interlingual pragmatic enrichment in translation. Journal of Pragmatics, 34, 1069-1089.

Setton, R. (1999). Simultaneous interpretation: a cognitive-pragmatic analysis. Amsterdam: John Benjamins.

Skelton, T., \& Valentine, G. (2003a). 'It feel like being Deaf is normal': an exploration into the complexities of defining D/deafness and young D/deaf people's indentities. The Canadian Geographer, 47(4), 451-466.

Skelton, T., \& Valentine, G. (2003b). Political participation, political action and political identities: young D/deaf people's perspectives. Space and Polity, 7(2), 117-134.

Skutnabb-Kangas, T. (1981). Bilingualism or not: the education of minorities. Clevedon: Multilingual Matters.

Smith, T. B. (1996). Deaf people in context. Unpublished PhD, University of Washington, Washington.

Sperber, D., \& Wilson, D. (1986). Relevance : communication and cognition. Oxford: Blackwell. 
Sperber, D., \& Wilson, D. (1995). Relevance : communication and cognition (2nd ed.). Oxford: Blackwell Publishers.

Spindler, G., \& Spindler, L. (1992). Cultural process and ethnography: an anthropological perspective. In M. D. LeCompte (Ed.), The handbook of qualitative research in education. London: Academic Press.

Spradley, J. P. (1979). The ethnographic interview. Fort Worth: Holt, Rinehart and Winston.

Squires, J. (2004). Equality and New Labour. Soundings: a journal of politics and culture, $27,74-85$.

Stolze, R. (2004). Creating "presence" in translation. In Claims, changes and challenges in translation studies selected contributions from the EST Congress (pp. 39 - 50).

Strauss, A., \& Corbin, J. (1990). Basics of qualitative research : grounded theory and procedures and techniques. London: SAGE Publications.

Supalla, T. (1986). The classifier system in American Sign Language. In C. Craig (Ed.), Noun classes and categorization. Philadelphia: John Benjamins.

Taylor, P. (Writer) (1998). Looking for Thomas... a search for my roots [Television]. In T. Riley (Producer), SeeHear. United Kingdom: BBC.

Tirkkonen-Condit, S. (1989). Theory and methodology in translation research. In S. Tirkkonen-Condit \& S. Condit (Eds.), Empirical studeis in translation and linguistics (pp. 3-18): University of Joensuu, Faculty of Arts.

Toury, G. (1978/revised 1995). The nature and role of norms in translation. In L. Venuti (Ed.), The translation studies reader. London: Routledge.

Venuti, L. (1998). The scandals of translation: towards an ethics of difference. London: Routledge.

Vermeer, H. J. (2000). Skopos and commission in tranlsational action. In L. Venuti (Ed.), The tranlation studies reader (pp. 221-232). London: Routledge.

Vuorinen, E. (1995). News translation as gatekeeping. In M. Snell-Hornby (Ed.), Translation as intercultural communication. Amsterdam: John Benjamins. Wilson, D., \& Sperber, D. (2002). Relevance Theory. Paper presented at the Working Papers in Linguistics 14, UCL. 
Woll, B. (2000). Exploring language, culture and identity: insights from sign language and the Deaf community. Paper presented at the Language Across Boundaries Conference, Anglia Polytechnic University, Cambridge. 